\title{
Extreme wave statistics of longcrested irregular waves over a shoal
}

\author{
Karsten Trulsen†, Anne Raustøl, Stian Jorde and Lisa Bæverfjord Rye
}

Department of Mathematics, University of Oslo, Norway

(Received $\mathrm{xx}$; revised $\mathrm{xx}$; accepted $\mathrm{xx}$ )

We report laboratory experiments of long-crested irregular water surface waves propagating over a shoal. For a sufficiently shallow shoal we find that the surface elevation can have a local maximum of skewness and kurtosis above the shallower part of the shoal close to the edge on the incoming side, and a local minimum of skewness over the downward slope on the lee side of the shoal. We find that the horizontal fluid velocity can have a local maximum and minimum of skewness at the same locations as those for the surface elevation. However, the kurtosis of the horizontal fluid velocity can have a local maximum over the downward slope on the lee side of the shoal, different from the location of the maximum of kurtosis of the surface elevation.

Key words: Authors should not enter keywords on the manuscript, as these must be chosen by the author during the online submission process and will then be added during the typesetting process (see http://journals.cambridge.org/data/relatedlink/jfmkeywords.pdf for the full list)

\section{Introduction}

Ocean surface waves propagating over non-uniform depth are subject to refraction (e.g. Massel 1996; Goda 2000). Their amplitude is governed by the energy flux conservation (e.g. Phillips 1977). For the special case of linear and long-crested waves normally incident on straight depth contours this leads to Green's law (Lamb 1932, Art. 185) stating that their amplitude will in the limit of very shallow water increase slightly and inversely proportional to the fourth root of the depth.

For linear gravity waves with wavenumber $k$ and angular frequency $\omega$ on water of depth $h$ the linear dispersion relation is $\omega^{2}=g k \tanh k h$, where $g$ is the acceleration of gravity, and the group velocity $c_{g}$ is

$$
\frac{c_{g} \omega}{g}=\frac{1}{2}\left(\tanh k h+k h \operatorname{sech}^{2} k h\right) .
$$

The graph of the non-dimensional group velocity is illustrated by the black curve in figure 1 . It has a local maximum of about 0.6 where $k h=\operatorname{coth} k h$ or $k h \approx 1.2$. For large depths the non-dimensional group velocity approaches 0.5 . For small depths the group velocity approaches zero asymptotically as $c_{g} \omega / g \approx k h$. Waves approaching a beach for small depths $k h<1.2$ will therefore have their amplitude slightly increased, while waves approaching shallower water from greater depths $k h>1.2$ will first have their amplitude decreased slightly and then increased slightly. At the same time the wavenumber will

$\dagger$ Email address for correspondence: karstent@math.uio.no 


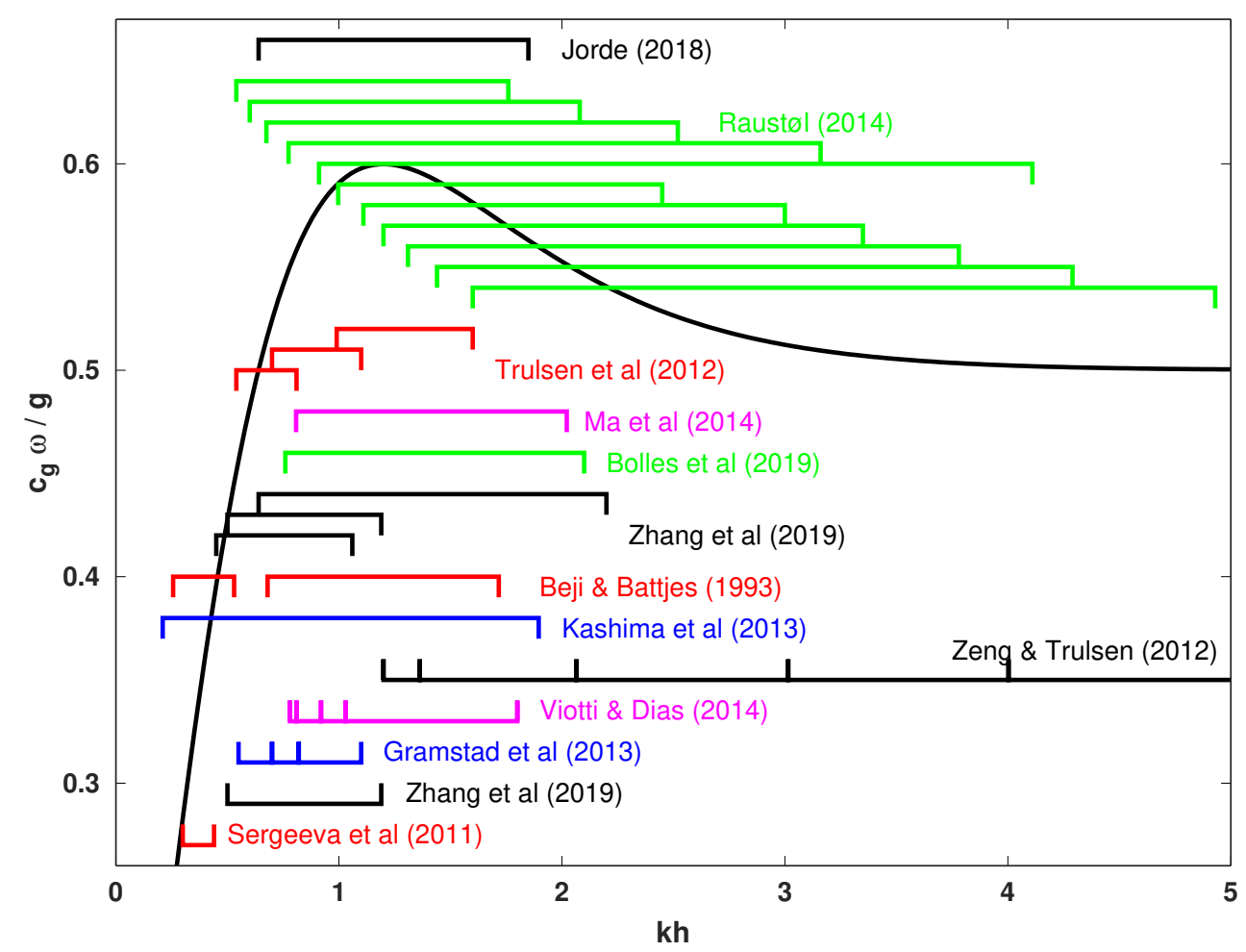

FIGURE 1. Group velocity indicated with solid curve (-). Experiments indicated by symbols $\square$ and simulations indicated by symbols $\sqcup$ with respect to the non-dimensional depth corresponding to the spectral peak frequency. Zeng \& Trulsen (2012) employed waves originating at rather deep water $k h=10$ not shown in this figure. All the mentioned works considered irregular wave statistics except Beji \& Battjes (1993) who only considered spectral changes.

increase slightly as the waves approach shallower water, therefore the steepness will also increase slightly.

The changes of depth, wavelength and amplitude induced by shoaling can modify the statistical distribution of the waves. This was investigated in the pioneering work of Bitner (1980) reporting a field experiment of waves propagating toward decreasing depth. A similar field experiment was also reported by Cherneva et al. (2005). In both cases they found noticeable deviation from Gaussian statistics.

When waves enter shallower water the spectral shape changes with the amplification of bound harmonics (e.g. Grue 1992; Beji \& Battjes 1993, 1994).

Recently it has been found that there can be a significant dynamic response that occurs after the depth change. This dynamic nonlinear behaviour was revealed in experiments as reported by Trulsen et al. (2012), Kashima et al. (2014), Ma et al. (2014), Bolles et al. (2019) and Zhang et al. (2019), and in numerical simulations as reported by Sergeeva et al. (2011), Zeng \& Trulsen (2012), Gramstad et al. (2013), Viotti \& Dias (2014), Majda et al. (2019) and Zhang et al. (2019). These recent studies suggest the existence of two different regimes: For waves that propagate onto a sufficiently shallow shoal it appears that a localised maximal concentration of extreme waves can occur at or near the shallow incident edge of the sloping bottom. For waves that propagate onto a sufficiently deep shoal it appears that no localised concentration of extreme waves occurs. Among the mentioned experimental and numerical works, Zeng \& Trulsen (2012) definitely belongs 

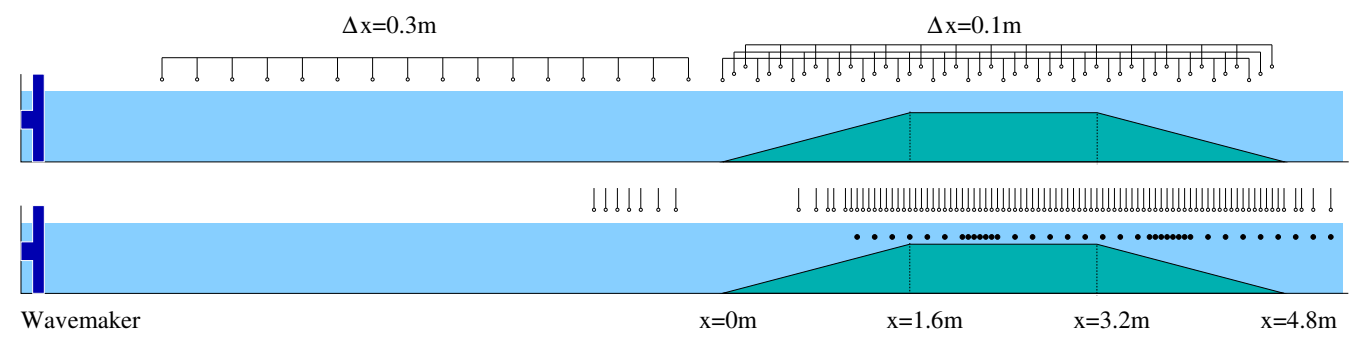

Figure 2. Setup of the two measurement campaigns. Upper part: First campaign (Raustøl 2014) with an array of 16 probes that was moved to four different locations such that the resolution before the shoal was $0.3 \mathrm{~m}$ and the resolution above the shoal was $0.1 \mathrm{~m}$. Lower part: Second campaign (Jorde 2018) with ADV placed at depth $48 \mathrm{~mm}$. The ultrasound probe locations are indicated by open circles $\circ$ in the air, while the ADV probe locations are indicated by filled circles $\bullet$ in the water. The wave maker is farther out to the left than indicated and the damping beach on the far right side is not shown.

to the deeper regime, while the deepest run reported by Trulsen et al. (2012) may belong to the deeper regime. However, for Zeng \& Trulsen (2012) the slope of the shoal is quite long and mild which may also have an influence.

Although recent work by Ducrozet \& Gouin (2017) suggests the enhancement of extreme wave statistics over a shoal is reduced for directional sea states, the early field measurements of Bitner (1980) and Cherneva et al. (2005) suggest such enhancement can occur under realistic field conditions.

The principal goal of this paper is to clarify the existence of, and transition between, the shallower and deeper regimes for long-crested waves propagating over a shoal in order to determine when an enhancement of extreme wave statistics can be expected. We also extend the consideration of extreme wave statistics to the fluid velocity field and discover that its statistics can be rather different from that of the surface elevation.

\section{Experimental setup}

The experiments were carried out in the $24.6 \mathrm{~m}$ long and $0.5 \mathrm{~m}$ wide tank at the Hydrodynamical Laboratory of the Department of Mathematics at the University of Oslo. At one end there is a wave maker and at the opposite end there is a damping beach. A shoal was installed with height $0.42 \mathrm{~m}$, with a linear up-slope of horizontal length $1.6 \mathrm{~m}$, a horizontal section of length $1.6 \mathrm{~m}$ and a linear down-slope of horizontal length $1.6 \mathrm{~m}$. The depth in the deeper part $h_{1}$ and the depth on top of the shoal $h_{2}$ are related by $h_{1}-h_{2}=0.42 \mathrm{~m}$. We align the $x$-axis along the tank with origin at the toe of the shoal (the part of the shoal closest to the wave maker). The distance from the equilibrium position of the wave maker to the toe of the shoal is estimated to be $10.78 \mathrm{~m}$. Surface elevation measurements were made with ultrasound probes from General Acoustics. Velocity measurements were made with a "Vectrino" Acoustic Doppler Velocimeter (ADV) from Nortek. The schematic view of the setup in figure 2 correctly indicates the positions of the probes relative to the shoal, but the wave maker is farther out to the left than indicated and the damping beach to the right is not shown.

An effort was made to ensure all corners of the shoal were rounded to avoid flow separation and turbulence generation.

The wave maker was programmed to generate a JONSWAP spectrum

$$
S(\omega)=\frac{\alpha g^{2}}{\omega^{5}} \mathrm{e}^{-\frac{5}{4}\left(\frac{\omega_{p}}{\omega}\right)^{4}} \gamma^{\exp \left(-\frac{1}{2}\left(\frac{\omega-\omega_{p}}{s \omega_{p}}\right)^{2}\right)}
$$




\begin{tabular}{|c|c|c|c|c|c|c|c|c|c|c|c|}
\hline \multirow[b]{2}{*}{ Run } & \multirow[b]{2}{*}{$T_{p}[\mathrm{~s}]$} & \multicolumn{5}{|c|}{ deeper side } & \multicolumn{5}{|c|}{ shallower side } \\
\hline & & $h_{1}[\mathrm{~cm}]$ & $k_{p} h$ & $H_{s}[\mathrm{~cm}]$ & $\epsilon$ & $U r$ & $h_{2}[\mathrm{~cm}]$ & $k_{p} h$ & $H_{s}[\mathrm{~cm}]$ & $\epsilon$ & $U r$ \\
\hline 1 & 1.1 & 50 & 1.8 & 1.4 & 0.018 & 0.0033 & 8 & 0.54 & 1.5 & 0.036 & 0.22 \\
\hline 2 & 1.0 & 50 & 2.1 & 1.6 & 0.024 & 0.0027 & 8 & 0.60 & 1.6 & 0.043 & 0.20 \\
\hline 3 & 1.1 & 53 & 2.5 & 2.5 & 0.030 & 0.00 & 11 & 0.65 & 2.5 & 0.052 & 0.19 \\
\hline 4 & 0.9 & 50 & 2.5 & 1.7 & 0.031 & 0.0019 & 8 & 0.68 & 1.6 & 0.048 & 0.16 \\
\hline 5 & 0.8 & 50 & 3.2 & 1.7 & 0.038 & 0.0012 & 8 & 0.78 & 1.5 & 0.052 & 0.11 \\
\hline 6 & 0.7 & 50 & 4.1 & 1.7 & 0.049 & 0.00071 & 8 & 0.91 & 1.4 & 0.058 & 0.078 \\
\hline 7 & 1.0 & 60 & 2.5 & 3.4 & 0.049 & 0.0033 & 18 & 0.97 & 3.1 & 0.060 & 0.064 \\
\hline 8 & 0.9 & 60 & 3.0 & 3.2 & 0.056 & 0.0021 & 18 & 1.1 & 2.9 & 0.063 & 0.046 \\
\hline 9 & 0.85 & 60 & 3.4 & 2.7 & 0.054 & 0.0014 & 18 & 1.2 & 2.5 & 0.058 & 0.033 \\
\hline 10 & 0.8 & 60 & 3.8 & 2.6 & 0.058 & 0.0011 & 18 & 1.3 & 2.3 & 0.059 & 0.027 \\
\hline 11 & 0.75 & 60 & 4.3 & 2.4 & 0.060 & 0.00076 & 18 & 1.4 & 2.1 & 0.059 & 0.020 \\
\hline 12 & 0.7 & 60 & 4.9 & 2.1 & 0.062 & 0.00052 & 18 & 1.6 & 1.8 & 0.058 & 0.014 \\
\hline
\end{tabular}

TABLE 1. Key parameters for all runs. The runs are numbered according to increasing dimensionless depth over the shoal. Run 3 belongs to the second campaign (Jorde 2018), the rest are from the first campaign (Raustøl 2014). The values of significant wave height $H_{s}$, steepness $\epsilon$ and Ursell number $U r$ are averages over all probes in front of or above the shoal, not including the probes at the edges of the sloping bottom.

with peak enhancement factor $\gamma=3.3$, peak frequency $\omega_{p}=\frac{2 \pi}{T_{p}}$ and the parameter $s=0.07$ for $\omega<\omega_{p}$ and $s=0.09$ for $\omega>\omega_{p}$. The wave amplitude was tuned by means of the parameter $\alpha$ to be as high as possible without visible wave breaking during the experiments.

Measurements were taken in two campaigns with two different probe arrangements and in 12 runs with different combinations of peak period $T_{p}$ and water depth. We have sorted these experimental runs by the dimensionless depth $k_{p} h$ on top of the shoal, where $k_{p}$ is the peak wavenumber derived from the peak period according to the linear dispersion relation for the relevant depth. The key parameters for the 12 runs are summarised in table 1. The steepness is $\epsilon=k_{p} a_{c}$ and the Ursell number is $U r=k_{p} a_{c} /\left(k_{p} h\right)^{3}$, where the characteristic amplitude is $a_{c}=\sqrt{2} \sigma$ and $\sigma$ is the standard deviation of the surface elevation. The significant wave height is $H_{s}=4 \sigma$.

In the first measurement campaign (Raust $\varnothing \mathrm{l} 2014$ ), with probe arrangement illustrated in the upper part of figure 2, eleven runs of only surface elevation measurements were made. An array of 16 ultrasound probes with spacing $0.3 \mathrm{~m}$ was moved to four different locations, allowing the surface elevation to be measured at a total of 64 positions with spatial resolution $0.3 \mathrm{~m}$ in front of the shoal and resolution $0.1 \mathrm{~m}$ above the shoal.

In the second measurement campaign (Jorde 2018) both surface elevation and horizontal velocity were measured at the positions indicated in the lower part of figure 2 . Only one run was made in this campaign, with water depth $h_{1}=0.53 \mathrm{~m}$ and peak period $T_{p}=1.1 \mathrm{~s}$, indicated as run 3 . The velocity measurements were made at depth $0.048 \mathrm{~m}$.

In the first campaign we had 16 ultrasound probes available. In the second campaign we had four ultrasound probes and one ADV available. In order to make all the "simultaneous" measurements indicated in figure 2 it was necessary to repeat each run of the first campaign four times, and to repeat the single run of the second campaign 43 times. In order to quantify the repeatability of the experiments we analyzed the measurements of the ultrasound probe at $x=-0.4 \mathrm{~m}$ of run 3 . For this probe position we have 35 repetitions, and we can compute the corresponding 35 repeated values of skewness and 
kurtosis. The coefficients of variation (the ratio of standard deviation over mean) of these 35 repeated values were found to be 0.0403 for the skewness and 0.00485 for the kurtosis. We are therefore confident that our experiments are sufficiently repeatable.

\section{Results}

In the following we present results obtained at each probe with respect to the horizontal position $(x)$ indicated along the first axis. The edges of the shoal at $x=0 \mathrm{~m}, 1.6 \mathrm{~m}, 3.2 \mathrm{~m}$ and $4.8 \mathrm{~m}$ are indicated by vertical dashed lines. Time averaging was used to compute the skewness $\gamma=\left\langle(\xi-\langle\xi\rangle)^{3}\right\rangle / \sigma_{\xi}^{3}$ and the kurtosis $\kappa=\left\langle(\xi-\langle\xi\rangle)^{4}\right\rangle / \sigma_{\xi}^{4}$, where $\sigma_{\xi}^{2}=\left\langle(\xi-\langle\xi\rangle)^{2}\right\rangle$ for $\xi$ being either the surface elevation or the horizontal velocity.

In figure 3 we show the intensity of the power spectral density in logarithmic scale. The spectra were computed with the Welch method. The maximum intensity is shown in white colour, weaker intensities are shown in darker colours. The positions along the tank where no measurements were taken are also shown in white colour. It is seen that the presence of the shoal provokes strongly enhanced higher harmonics, in agreement with previous work (Grue 1992; Beji \& Battjes 1993, 1994), provided the shoal is sufficiently shallow. We observe that the appearance of the higher harmonics is part of a dynamic process that has it maximum intensity a short distance inside the shallower part of the shoal and then relaxes.

In figure 4 we show the skewness of the surface elevation. The skewness can have a local maximum some distance inside the shallower part of the shoal and can have a local minimum some distance onto the down-slope on the lee side. There is a tendency that the location of the maximum skewness approaches the incident edge of the shallower part of the shoal with increasing depth of the shoal.

In figure 5 we show the kurtosis of the surface elevation. The kurtosis can also have a local maximum some distance inside the shallower part of the shoal, but does not have a local minimum some distance into the lee side. The locations of the maxima of the kurtosis and the skewness appear to coincide, including the tendency that the location of the maximum approaches the incident edge of the shallower part of the shoal with increasing depth of the shoal.

In figure 6 we show a comparison between the statistics of the surface elevation and of the horizontal velocity for run 3 . The skewness of the surface elevation and the skewness of the horizontal velocity follow each other. However, for the kurtosis the behaviour of the surface elevation and of the horizontal velocity is rather different. The horizontal velocity does not have a maximum of kurtosis on top of the shoal, but has a local maximum on the lee side.

\section{Discussion}

Our experiments seem to confirm the existence of two regimes for wave statistics when long-crested waves propagate over a shoal. When the shoal is shallower than some threshold depth the skewness and the kurtosis can be significantly enhanced over the shallower part of the shoal while the skewness can have a local minimum on the lee side of the shoal. The maximum of the skewness and the kurtosis is achieved some distance into the shallower part of the shoal, moving closer to the incident edge of the shallower part of the shoal as the depth the shoal increases.

The power spectrum shows strongly enhanced higher harmonic behaviour at the same locations where the skewness and the kurtosis of the surface elevation are amplified. The enhanced higher harmonic behaviour also depends on the shoal being sufficiently shallow, 

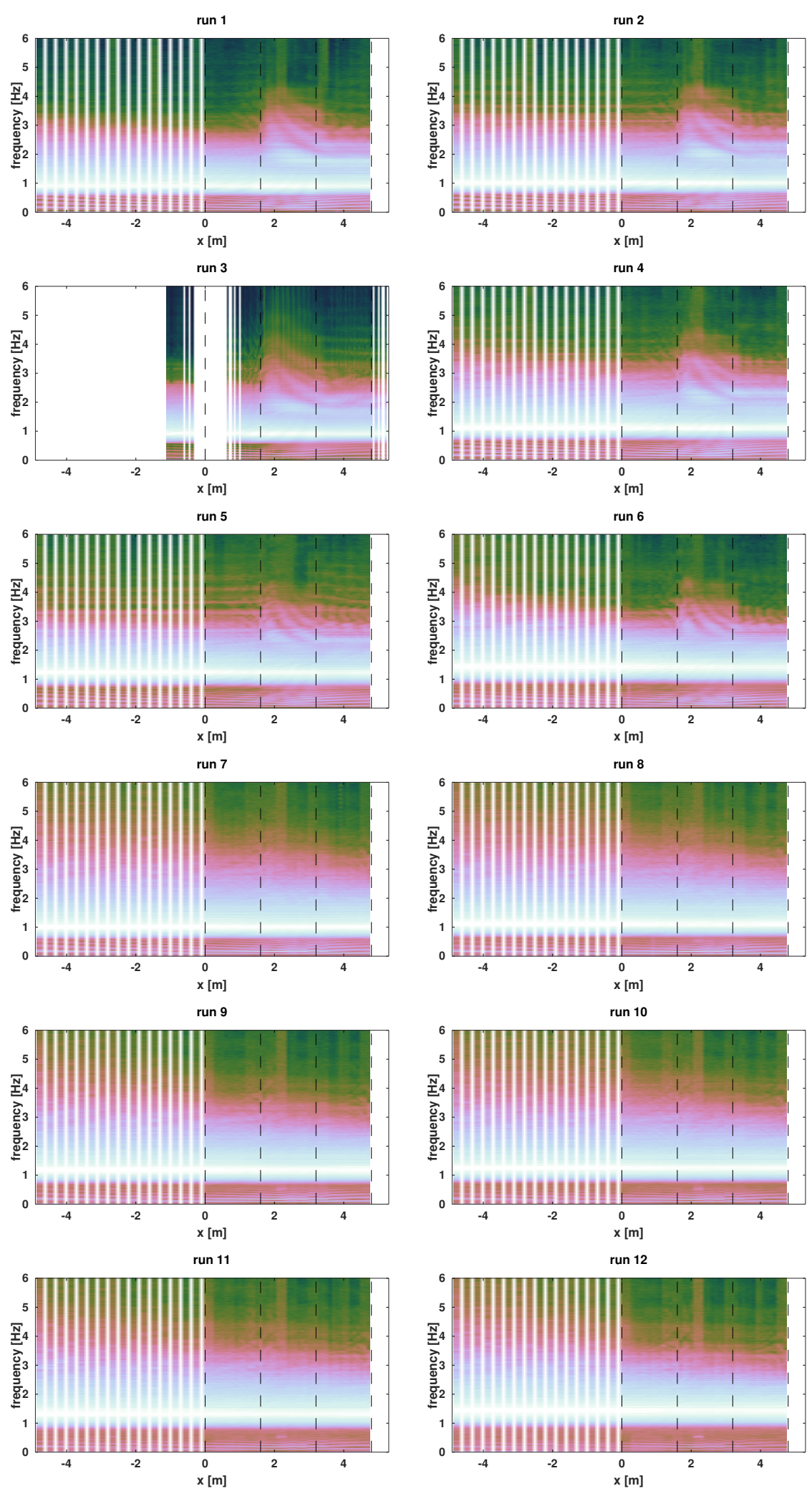

Figure 3. Intensity of power spectral density of the surface elevation. White colour indicates maximum power or lack of measurement. For enhanced visibility the thickness of the each spectrogram is doubled for the probes in front of the shoal $x<0$. 

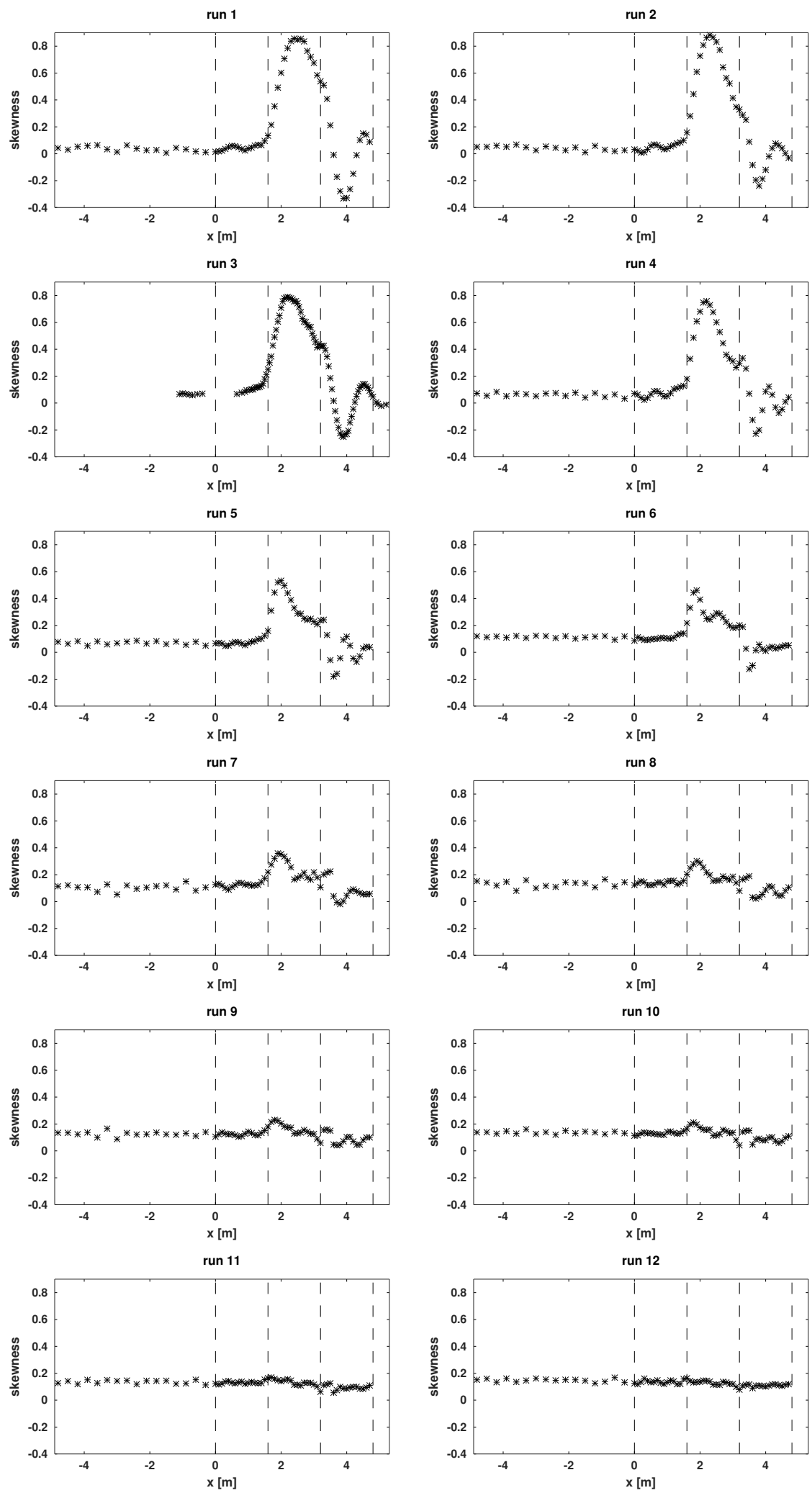

Figure 4. Skewness of surface elevation. 

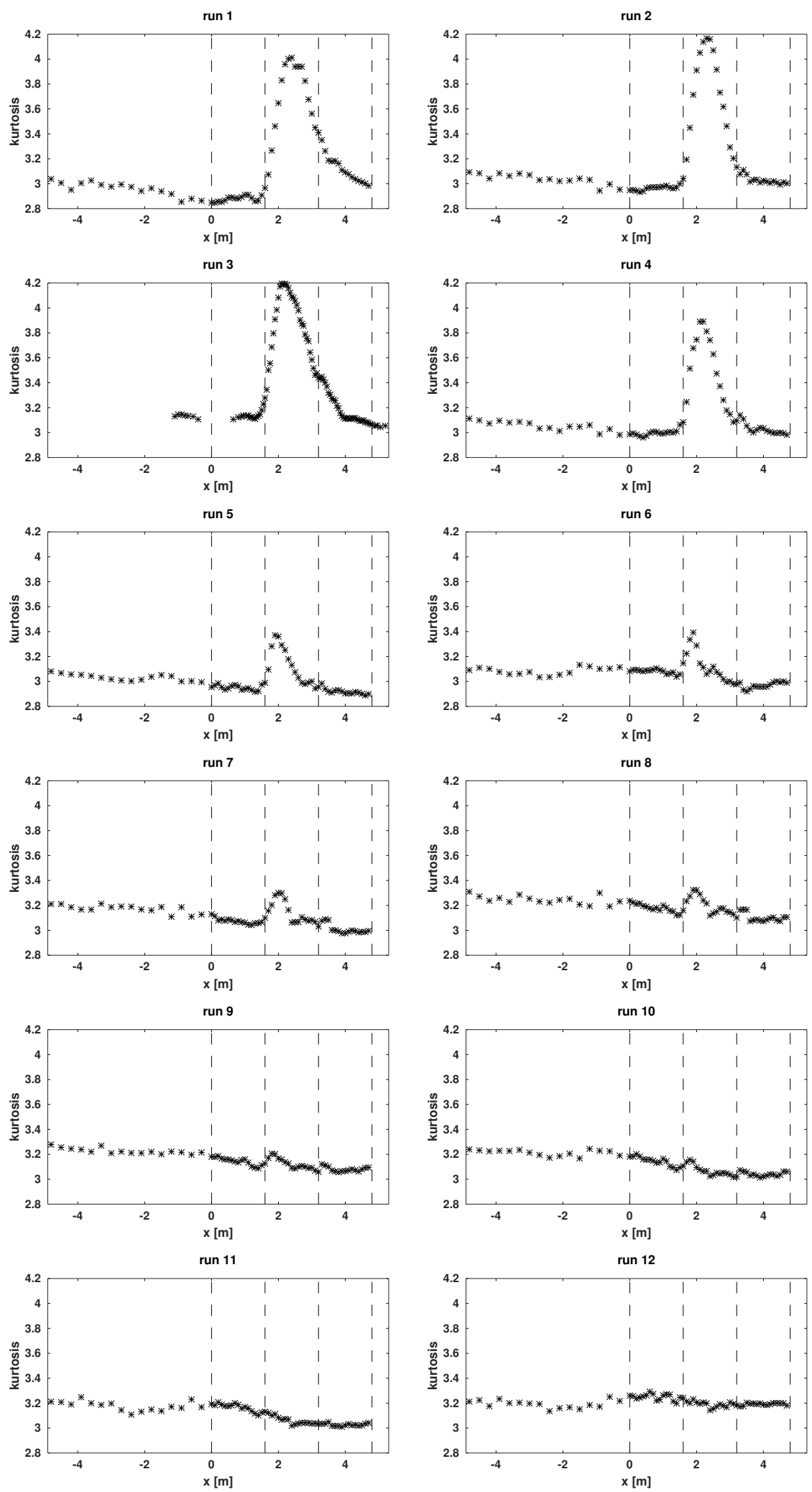

Figure 5. Kurtosis of surface elevation. 

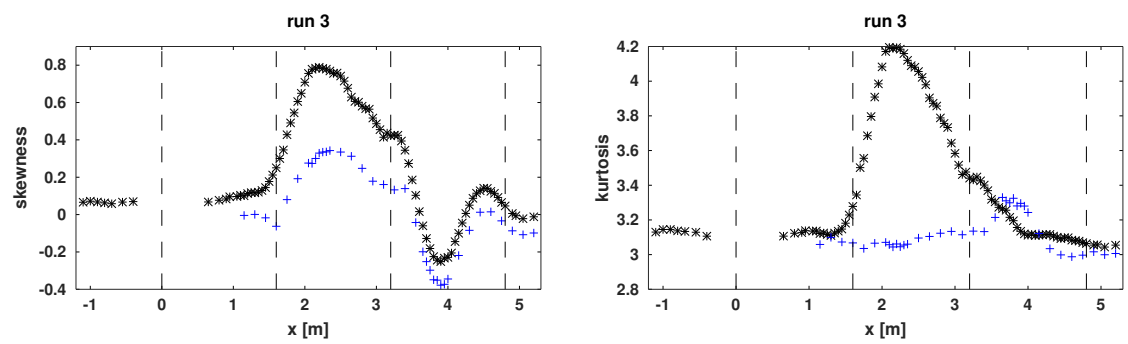

Figure 6. Skewness (left) and kurtosis (right) of surface elevation $(*)$ and horizontal velocity $(+)$.

our results seem to suggest the threshold depth for enhanced higher harmonics may be smaller than the threshold depth for enhanced extreme wave statistics.

Our results suggest that the threshold depth for enhanced extreme wave statistics may be close to $k h=1.3$ for the shallower part of the shoal, however, it should be anticipated that this may depend on the steepness and the bandwidth of the waves and on the slope of the shoal.

The behaviour of the kurtosis of the horizontal velocity is surprising, with the maximum achieved on the lee side rather than above the shallower part of the shoal. Recently, we have carried out numerical simulations showing the same behaviour as that seen in figure 6 , these simulations will be published in the future.

As far as giving an explanation for the anomalous behaviour of the skewness and the kurtosis is concerned, we suspect it is not due to flow separation around the sharp edges of the shoal since an effort was made to round the corners to avoid separation. We also suspect it is not due to wave breaking provoked by the shoal since an effort was made to reduce the amplitude such that breaking did not occur. Our best guess is that the phenomenon is related to a relaxation process: When a wave field enters a new environment the process of reaching a new equilibrium state can give extreme excursions in the values of certain statistical parameters.

\section{Conclusion}

We have carried out laboratory experiments of long-crested irregular water surface waves propagating over a shoal. For a sufficiently shallow shoal we find that the surface elevation can have a local maximum of skewness and kurtosis above the shallower part of the shoal close to the edge on the incoming side, and a local minimum of skewness over the downward slope on the lee side of the shoal. We also find that the horizontal fluid velocity can have a local maximum and minimum of skewness at the same locations as those for the surface elevation. The kurtosis of the horizontal fluid velocity can have a local maximum over the lee side of the shoal different from the location of the maximum of kurtosis of the surface elevation.

\section{Dedicated to Kristian B. Dysthe on the occasion of his 80th birthday.}

We thank Olav Gundersen for building and installing the experimental setup, Atle Jensen for assistance with the experiments, and Harald E. Krogstad for valuable feedback. This work has been supported by the Research Council of Norway (RCN) through grants 214556 and 256466. 


\section{REFERENCES}

BejI, S. \& Battues, J. A. 1993 Experimental investigation of wave propagation over a bar. Coastal Engineering 19, 151-162.

Beji, S. \& BAtTues, J. A. 1994 Numerical simulation of nonlinear wave propagation over a bar. Coastal Engineering 23, 1-16.

Bitner, E. M. 1980 Non-linear effects of the statistical model of shallow-water wind waves. Appl. Ocean Res. 2, 63-73.

Bolles, C. T., Speer, K. \& Moore, M. N. J. 2019 Anomalous wave statisics induced by abrupt depth change. Phys. Rev. Fluids 4, 011801.

Cherneva, Z., Petrova, P., Andreeva, N. \& Guedes Soares, C. 2005 Probability distributions of peaks, troughs and heights of wind waves measured in the Black Sea coastal zone. Coastal Engineering 52, 599-615.

Ducrozet, G. \& Gouin, M. 2017 Influence of varying bathymetry in rogue wave occurrence within unidirectional and directional sea-states. J. Ocean Eng. Mar. Energy 3, 309-324.

Goda, Y. 2000 Random seas and design of maritime structures. World Scientific, 443 pp.

Gramstad, O., Zeng, H., Trulsen, K. \& Pedersen, G. K. 2013 Freak waves in weakly nonlinear unidirectional wave trains over a sloping bottom in shallow water. Phys. Fluids 25, 122103 .

Grue, J. 1992 Nonlinear water waves at a submerged obstacle or bottom topography. J. Fluid Mech. 244, 455-476.

Jorde, S. 2018 Kinematikken i bølger over en grunne. Master's thesis, University of Oslo.

Kashima, H., Hirayama, K. \& Mori, N. 2014 Estimation of freak wave occurrence from deep to shallow water regions. Coastal Engineering Proceedings 1 (34), 36.

Lamb, H. 1932 Hydrodynamics. Dover, 738 pp.

MA, Y., Dong, G \& MA, X. 2014 Experimental study of statistics of random waves propagating over a bar. Coastal Engineering Proceedings 1 (34), 30.

Majda, A. J., Moore, M. N. J. \& QI, D. 2019 Statistical dynamical model to predict extreme events and anomalous features in shallow water waves with abrupt depth change. Proc. Natl. Acad. Sci. 116, 3982-3987.

Massel, S. R. 1996 Ocean Surface Waves: Their Physics and Prediction. World Scientific, 491 $\mathrm{pp}$.

Phillips, O. M. 1977 The dynamics of the upper ocean.. Cambridge University Press.

RAustøL, A. 2014 Freake bølger over variabelt dyp. Master's thesis, University of Oslo.

Sergeeva, A., Pelinovsky, E. \& Talipova, T. 2011 Nonlinear random wave field in shallow water: variable Korteweg-de Vries framework. Nat. Hazards Earth Syst. Sci. 11, 323-330.

Trulsen, K., Zeng, H. \& Gramstad, O. 2012 Laboratory evidence of freak waves provoked by non-uniform bathymetry. Phys. Fluids 24, 097101.

Viotti, C. \& Dias, F. 2014 Extreme waves induced by strong depth transitions: Fully nonlinear results. Phys. Fluids 26, 051705.

Zeng, H. \& Trulsen, K. 2012 Evolution of skewness and kurtosis of weakly nonlinear unidirectional waves over a sloping bottom. Nat. Hazards Earth Syst. Sci. 12, 631-638.

Zhang, J., Benoit, M., Kimmoun, O., Chabchoub, A. \& Hsu, H.-C. 2019 Statistics of extreme waves in coastal waters: Large scale experiments and advanced numerical simulations. Fluids 4 (99), 1-24. 